\title{
Electron-microscope evidence for intramicrovillous fat absorption by the small intestinal epithelium of rats
}

\author{
MARGOT SHINER \\ From the Gastroenterology Research Unit, Central Middlesex Hospital, London, \\ and the Electron Microscope Unit, Department of Zoology and Comparative Anatomy, \\ St. Bartholomew's Hospital Medical College, London
}

EDITORIAL SYNOPSIS This paper demonstrates by electron microscopy that large numbers of droplets thought to be fat can pass through the microvilli of the intestinal mucosal cell.

After feeding highly unsaturated oils either to humans or animals, fat is rarely seen in the region of the brush border of the intestinal epithelium when this area is studied by electron microscopy. On the other hand, fat droplets are easily observed within the endoplasmic reticulum and Golgi apparatus of these cells shortly afterwards.

Two pathways for the entry of fat have been observed in humans and animals. The first, and most widely favoured, proposes entry by pinocytosis (Palay and Karlin, 1959; Palay and Revel, 1964; Ladman, Padykula, and Strauss, 1963; Ashworth, Stembridge, and Sanders, 1960). Here the fat droplets, advancing from the lumen of the intestine, find their way between the microvilli, and, on reaching the surface membrane of the cell, are then engulfed by the membrane, which is nipped off from its continuous sheath and becomes part of the endoplasmic reticulum (Fig. 1). The droplets observed in the spaces between the microvilli had an average diameter of $500 \AA$ or less (Palay and Revel, 1964). The second theory favours the carriage of fat droplets within the microvilli (Lacy and Taylor, 1962). These droplets have been demonstrated close to the inner of the double membranes surrounding the microvilli, facing the interior of the microvilli. Their size was described as up to $250 \AA$. Such entry of fat would imply that droplets were transported through the lipid-protein membrane instead of being engulfed by membrane. The membranes, clearly demonstrated around fat droplets in the endoplasmic reticulum, would thus have to be derived from intracellular sources (Sjöstrand, 1963). Unfortunately fat is even more rarely observed in the space immediately below the microvilli, the terminal web, so that evidence of continuity of (a)

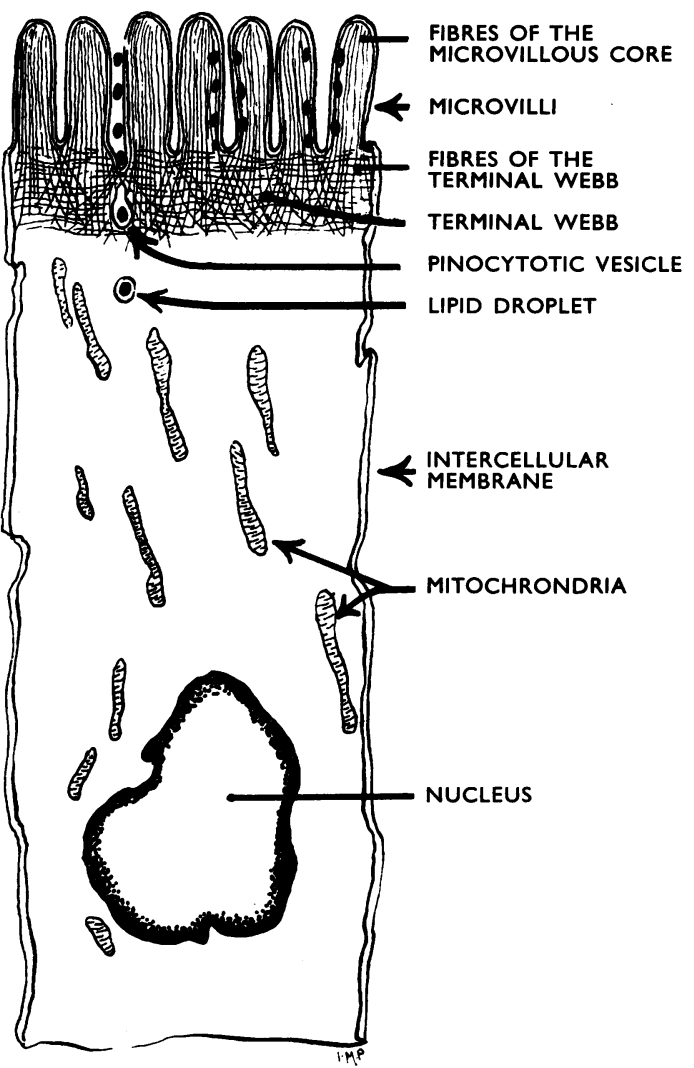

FIG. 1. Diagram of an intestinal epithelial cell showing entry of fat droplets (a) by pincocytosis, (b) by intramicrovillous transport. In (a) the droplets are carried between the microvilli. 
movement of fat particles from the lumen of the intestine to the point of their appearance in the endoplasmic reticulum of the absorbing cell is almost entirely lacking. Support for the intramicrovillous carriage of fat, possibly in the form of micelles, has also been advanced by Rostgaard and Barrnett (1964). Their particles were about 40 to $50 \AA$ in diameter.

This communication presents further evidence to show that large numbers of droplets, presumed to be fat, can pass into the microvilli and supports the view that the microvilli may be the vehicle for transport of fat from the lumen of the intestine to the interior of the absorbing cell.

\section{METHOD AND MATERIAL}

White, adult, female Wistar rats, weighing approximately $250 \mathrm{~g}$., were starved for 36 hours, only water being allowed. During that time coprophagy was prevented as much as possible by housing the animals in wire mesh cages. On the morning of the experiment two rats were anaesthetized intraperitoneally with $0.25 \mathrm{ml}$. $(15 \mathrm{mg}$.) of nembutal diluted to one half with sterile water. The abdomen was opened and in rat 1 , acting as control, $0.3 \mathrm{ml}$. of $3 \%$ glutaraldehyde-cacodylate fixative (Sabatini, Bensch, and Barrnett, 1963) at $p \mathrm{H} 7.4$ was injected into the lumen of the jejunum one minute before excising a ring of jejunum about $5 \mathrm{~mm}$. long just beyond the duodeno-jejunal flexure.

In rat 2, after opening the abdomen, $0.5 \mathrm{ml}$. of linseed oil was injected into the duodenum through a needle and syringe. Exactly five minutes later glutaraldehyde fixation of the jejunum was begun as in rat 1 , and a ring of upper jejunum was excised one minute later.

The excised tissues were immediately fixed in a $3 \%$ glutaraldehyde-cacodylate mixture at $4^{\circ} \mathrm{C}$. for six hours. Each ring was cut into 1-2 $\mathrm{mm}$. squares soon after fixation. The tissues were washed in a cacodylate-sucrose mixture overnight and then postfixed in a $1 \%$ phosphate buffered osmium solution at $p \mathrm{H} \mathbf{7 \cdot 3}$ for two hours at $4^{\circ} \mathrm{C}$. Dehydration through increasing concentrations of ethanol was followed by Epon embedding using Luft's mixture A and B (Luft, 1961) in 40/60 proportions. The blocks were cut on a Porter-Blum MT-1 ultramicrotome. The sections were stained with uranyl acetate (Watson, 1958) followed by lead citrate (Reynolds, 1963) diluted to 1 . The stained grids were examined with a Siemens E.M.I. electron microscope.

\section{PREVIOUS ELECTRON-MICROSCOPE OBSERVATIONS}

In our first publication on the subject of fat transport across the brush border of human small intestinal absorbing cells (Shiner, Lacy, and Hudson, 1962), we were unable to demonstrate fat uptake by the brush border, despite clear evidence of fat uptake by the endoplasmic reticulum in the interior of the cell. The brush border of the intestinal epithelium was continuously studied over the following number of years in both human and rat tissue after feeding fat. Various oils, such as olive oil, corn oil, codliver oil, and linseed oils were administered in their natural state, always into the duodenum. Jejunal tissue was obtained by excision or biopsy, from five to 60 minutes after oil instillation. Monoglyceride fat was also given to humans and rats. A number of observations were made which have proved helpful:

1 Entry of fat from the lumen of the cell may be a very rapid process, taking seconds rather than minutes.

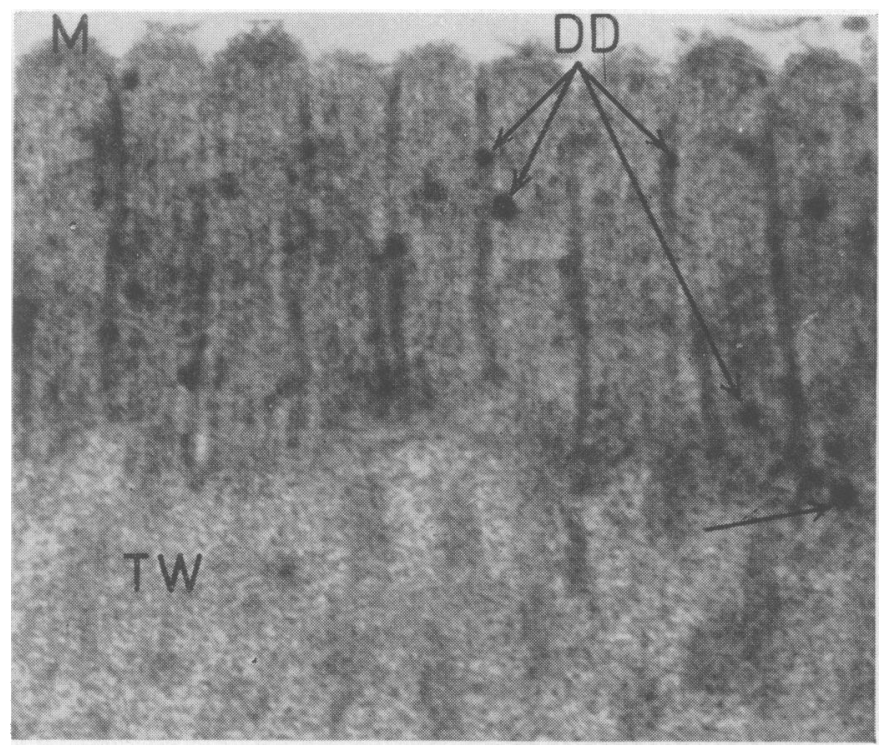

FIG. 2. Electron micrograph of the apical part of a villous epithelial cell in the upper jejunum of rat 2 after linseed oil instillation. The microvilli $(M)$ are shown in longitudinal section. Numerous small and large dense droplets (DD), assumed to be fat, 100-300 $A$ in diameter, are seen within the lateral membranes of the microvilli or facing the interior of the microvilli. One of these droplets (arrow) is seen in the region of the terminal web $(T W) . \times 30,000$ 
2 Oils in unemulsified form can be absorbed.

3 Convincing evidence for pinocytosis was not observed.

4 Demonstrable droplets in the region of the microvilli and the terminal web were a rare event.

Nevertheless the impression was gained that tiny round droplets of the order of $100 \AA$ or less seemed to settle in large numbers on the membranes of the microvilli and were seen better in cross sections of microvilli than in longitudinal sections. For various technical reasons, chiefly because methacrylate embedding was used and the sections were unstained, the quality of the micrographs obtained was never sufficiently good to present convincing evidence of droplet absorption or adsorption by the microvilli. The minute droplets which studded the microvilli could be traced to the region of the terminal web where they were not surrounded by membrane.

PRESENT ELECTRON MICROSCOPIC OBSERVATIONS

Examination of the absorbing cells of the control animal (rat 1) under the conditions described proved that the technical quality of the tissue was satisfactory and that the brush border membranes were smooth and devoid of droplets. There were no dense droplets in the interior of these cells. Indeed, of many blocks of rat 2, given linseed oil, similar observations could be made of the brush border, though intracellular dense droplets of large size ( $500 \AA$ or more) could usually be seen. However, in rat 2 areas could be found where the brush border, showing microvilli in either longitudinal, oblique, or cross section, contained large numbers of particularly densely staining droplets. These are shown in Figures 2 to 6.

Figure 2 demonstrates the relationship of these droplets to longitudinally sectioned microvilli. Of particular interest is the position of the smaller dense particles within the double membranes along the sides of the microvilli. One of these is seen to enter the terminal web.

Figure 3 represents an oblique section through the apical region of three or four villous cells. The microvilli occupy the upper part of the micrograph. Nearly every one of these contains one or more densely staining droplets. Immediately below the microvilli the fibres of the terminal web can be seen in oblique section. This region contains only a few droplets of similar density to those seen in the microvilli. Several large dense droplets can be identified within the endoplasmic reticulum of the lower two cells and a few smaller droplets are seen close to or within the intercellular membranes.

Figure 4 represents a magnification of part of the brush border shown in Figure 3. It demonstrates more clearly the position of the dense droplets within the microvilli.
Figure 5 shows a higher magnification of microvilli in oblique section. In this micrograph the larger sized droplets appear to predominate. They mainly occupy the core of the microvilli. Two large dense droplets are clearly identified in the area of the terminal web in close relation to its fibres. These droplets do not appear to be surrounded by membrane.

Figure 6 shows the microvilli in near circular section from an area of the brush border of Figure 3. Of great interest are the large numbers of smaller sized droplets situated between the double membranes of the microvilli. Some of these appear to be wedged in the space between the membranes which measures between 93.2 and 111.2 $\AA$ (Millington, 1964).

In all micrographs numerous dense droplets varying in size from about 100 to $300 \AA$ are seen in the region of the microvilli and a few large droplets of around $500 \AA$ are found in the area of the terminal web. These latter particles do not appear to be surrounded by membrane. The droplets studied in relation to the microvilli occupied positions within the double membrane surrounding the microvilli, or attached to the inner of the two membranes and bulging into the interior of the microvilli. Very few droplets were found inside the microvilli, seemingly unattached to the membrane, or in the spaces between microvilli (Fig. 5).

A great variation in droplet size was observed. About equal numbers of droplets of smaller and larger size were found. The smaller droplets were always arranged between the two membranes of the microvilli. As also observed in the intercellular membranes of the epithelium, there appeared to be a close relationship between dense droplets, assumed to be fat, and membranous structures.

The droplets found within the microvilli appeared enmeshed in the fibres of the microvillous core rather than occupying the space between the core and the inner of the two membranes (McNabb and Sandborn, 1964). The fibres of the central core and the terminal web appeared as paired structures (Millington and Finean, 1962).

The few droplets observed in the region of the terminal web were in close contact with the fibres of the terminal web.

The droplets themselves were circular or oval and had a homogenous density, but occasionally denser and small bodies could be found within a larger droplet. Their free surfaces facing the interior of the microvilli were not surrounded by membrane and therefore their entry by membranous invaginations was not evident. One or more droplets could be seen in any one plane in relation to a single microvillus. 


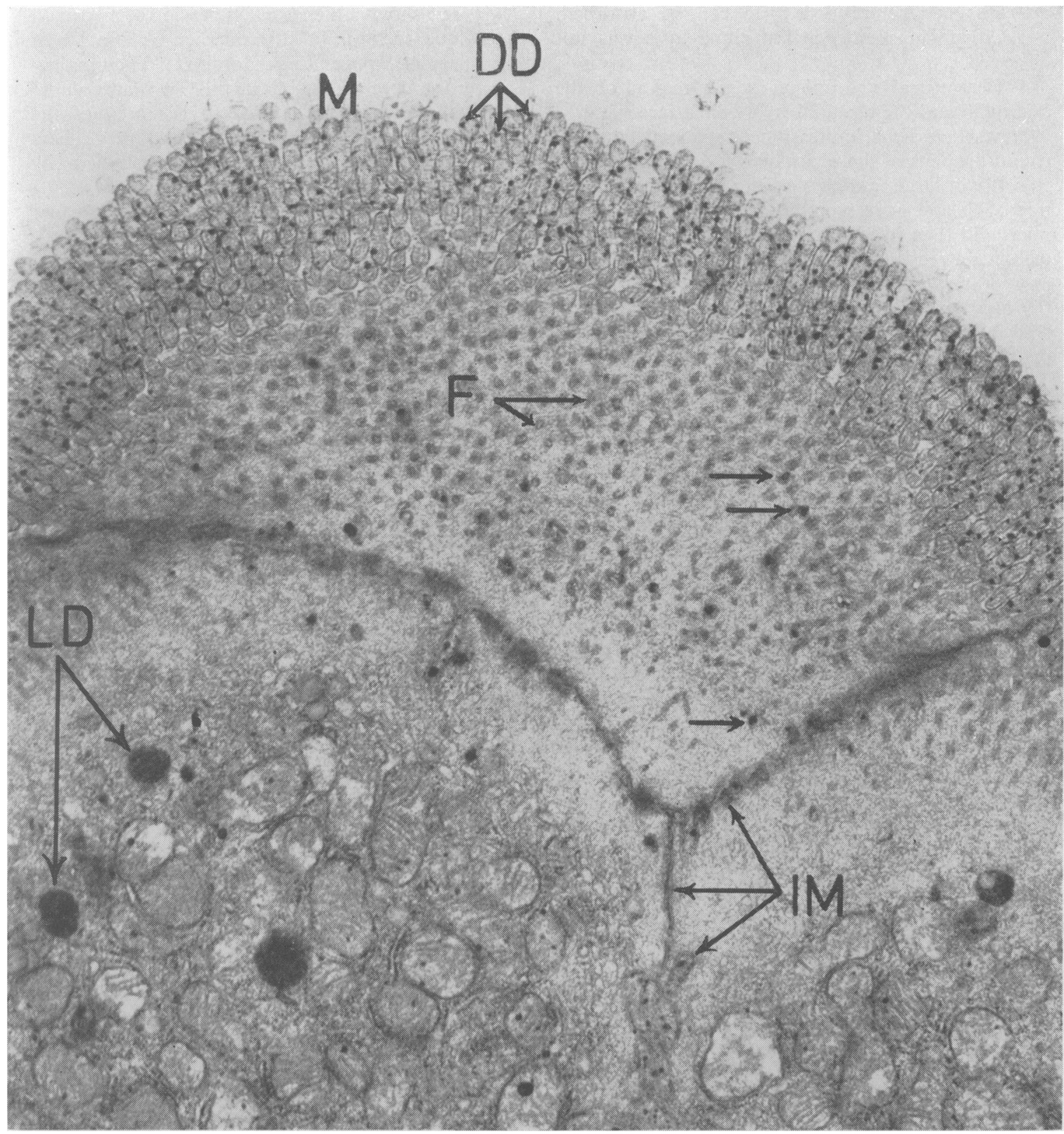

FIG. 3. Electron micrograph showing the apical parts of three or four epithelial cells in oblique section from the upper jejunum of rat 2 after linseed oil instillation. Large numbers of dense droplets $(D D)$ are seen within the microvilli $(M)$. Only a few droplets of similar density are seen in the area of the terminal webb (arrow), closely related to the fibres $(F)$. Numerous smaller dense droplets, also assumed to be fat, are lying close to the intercellular membranes (IM) of the cells. Large droplets $(L D)$ are found within the cytoplasm of the lower two cells. $\times 20,000$. 


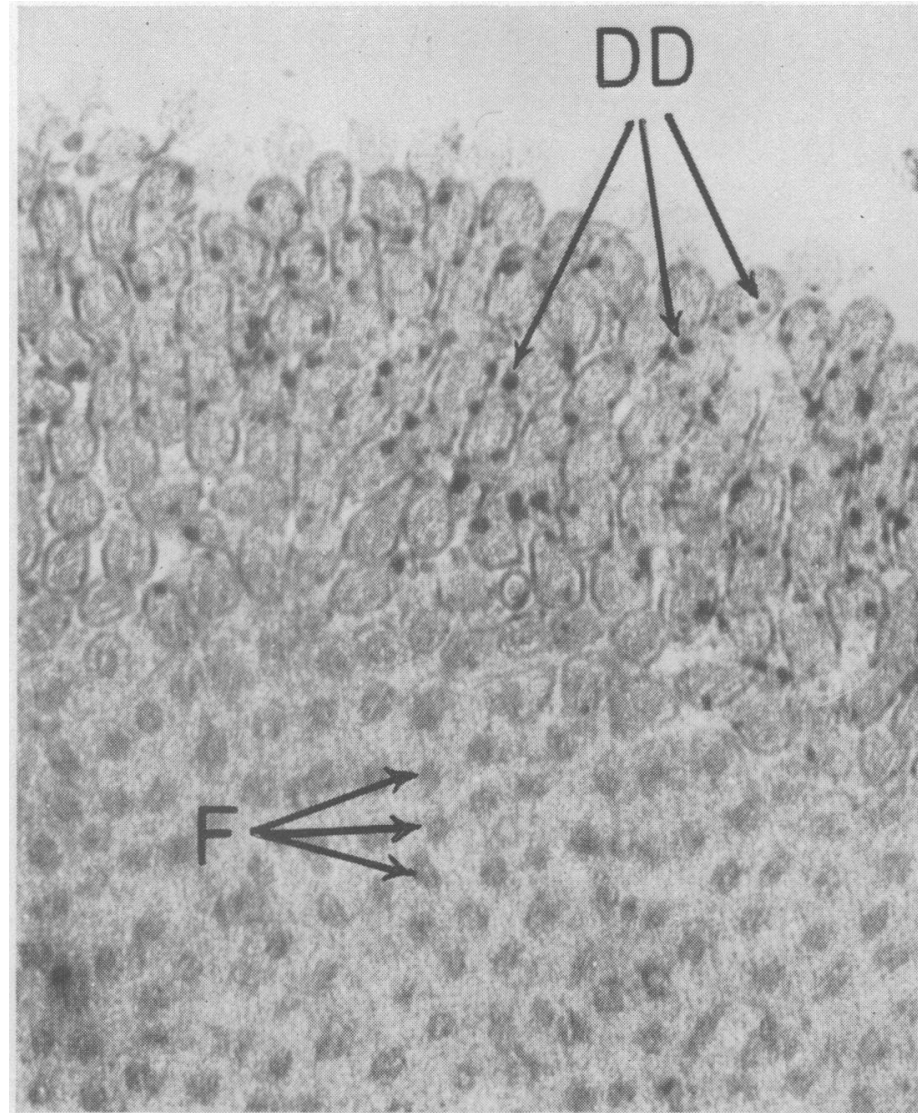

FIG. 4.

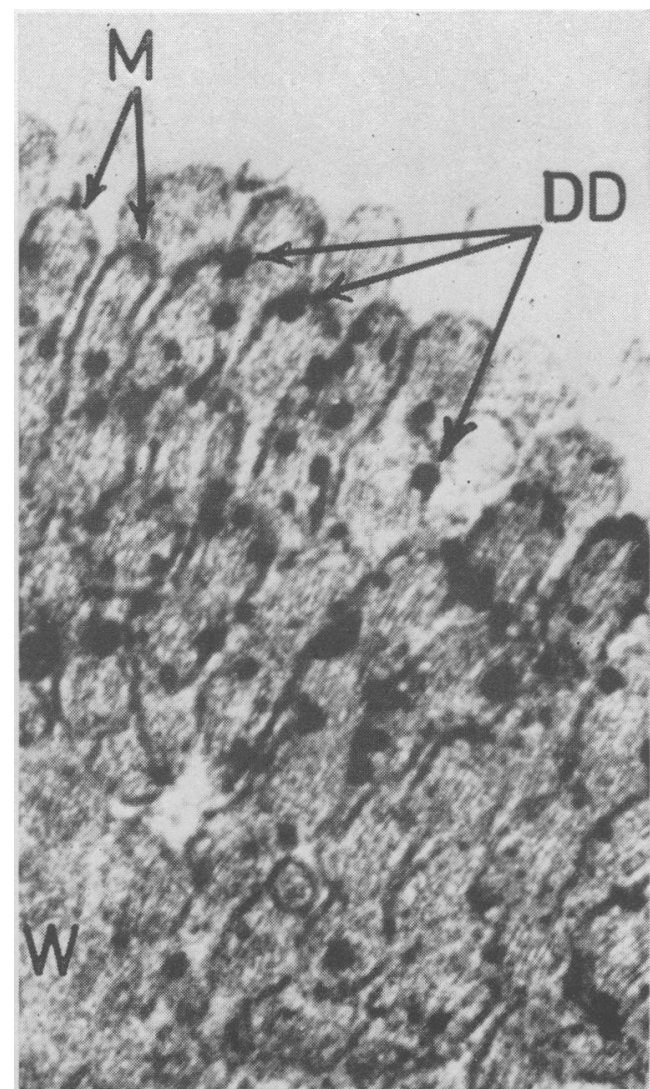

FIG. 5.

FIG. 4. Higher magnification of part of the brush border of Fig. 3 showing more clearly the arrangement of dense droplets $(D D)$ within the microvilli $(M)$ and the fibres $(F)$ of the terminal web. $\times 30,000$.

FIG. 5. Higher magnification of part of the brush border of Fig. 3, showing microvilli $(M)$ in oblique section. Large dense droplets $(D D)$ predominate and are situated mainly in the core of the microvilli. $\times 60,000$.

\section{DISCUSSION AND CONCLUSIONS}

Evidence has been submitted to show that droplets, assumed to be fat, can enter through the membranes of the microvilli and can be carried either along the membranes or in the interior of the microvillus to the main cell without the requirement of a pinocytotic mechanism. How these droplets diffuse through the microvillous membranes and in what chemical form is not known at present. That lipid can apparently diffuse through the lipid-protein structure of membranes can also be observed when fat droplets leave the cell through the intercellular membranes or through the basal membrane of the cell.

Relating the diameter of the dense droplets described to their chemical composition, it is to be noted that the size of the droplets is larger than micelles which are probably no bigger than $40 \AA$ (Borgström, 1964). As to the chemical form of the droplets at the point of membrane transport, it is possible that the droplets seen may be aggregations of molecules which arrived at the membrane in more dispersed form or some re-esterification of fatty acids may have occurred. Although biochemical evidence exists against the latter hypothesis it cannot at present be conclusively excluded. Present biochemical thinking favours the first possibility. In either event it is unlikely that particulate absorption and transport of lipids across the microvillous membrane takes place, since in that event oblique or cross sections of the microvillous area should show at least some droplets attached to the micro- 


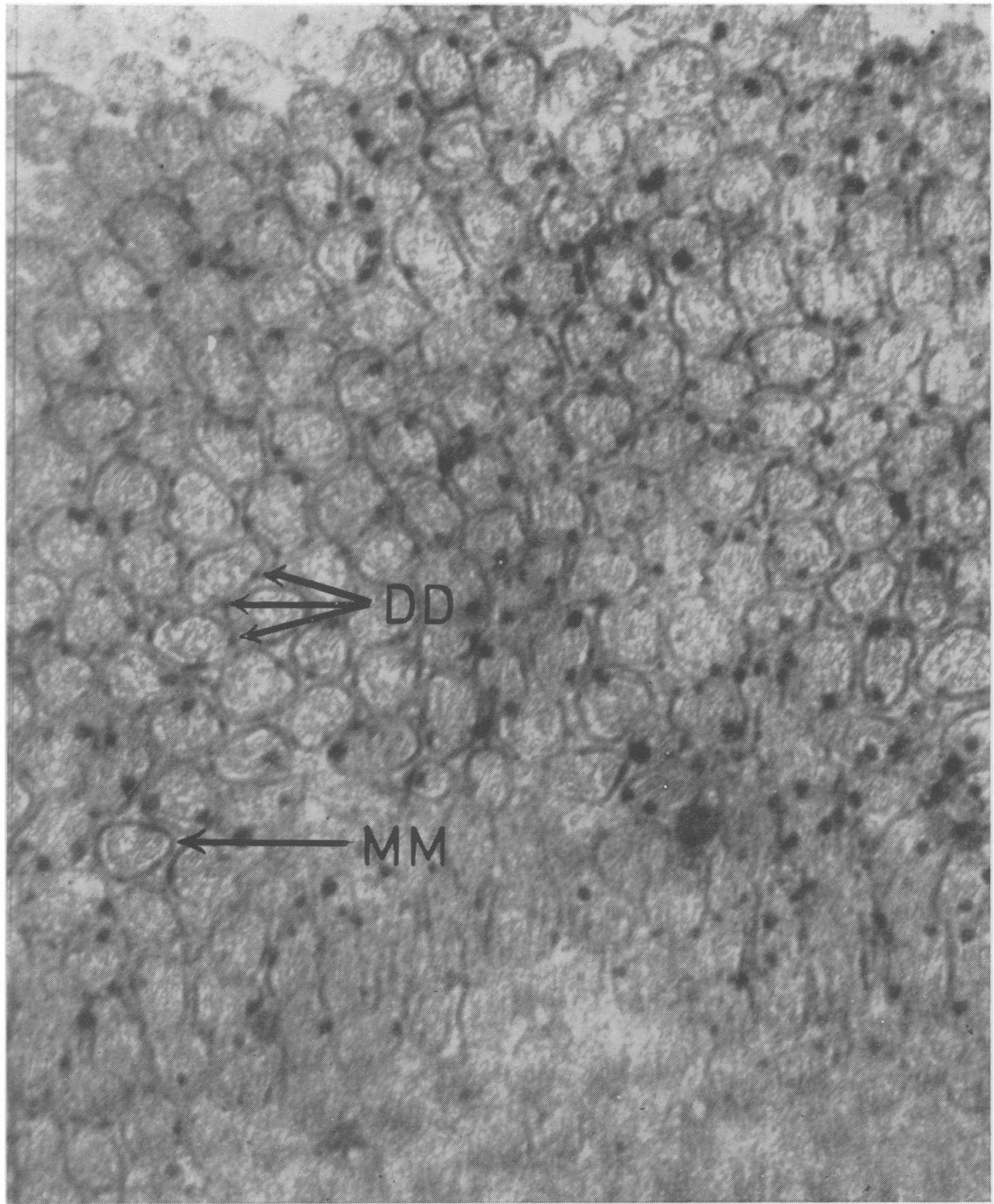

FIG. 6. Higher magnification of part of the microvillous region of Fig. 3. Of particular interest are the smaller sized dense droplets (DD) arranged within the microvillous membranes (MM). Many of the larger droplets face the interior of the microvilli shown here in cross section. $\times 60,000$. 
villous membranes with their free border facing outwards rather than into the interior of the microvilli. This is not apparent from the appearances in the micrographs presented.

Finally it seems important to discuss the quantitative significance of intramicrovillous transport versus pinocytotic entry of fat droplets. The observed speed with which fat enters the epithelial cell would favour intramicrovillous transport since the total area for absorption by each cell is enormously increased by the microvilli if these are thought of as part of the cell. It has been estimated that if pinocytosis were the main physiological way for entry of fat the number of pinocytotic vesicles and the rate at which they are formed should be very large and rapid and therefore require a high expenditure of energy (Parsons, 1963). Yet the actual pinocytotic vesicles shown to contain a fat droplet are few. On the other hand the activation energy required for the passage of at least single molecules of lipid from a watery medium into the lipid interior of the membrane may be low and therefore the rate of entry high (Danielli, 1964). The entry of lipid by simple diffusion may not be the only mechanism of transport and, as suggested by Danielli, there may be a specific receptor mechanism at the surface of the cell membrane.

Transport of lipids across cell membranes remains primarily a biochemical problem. But electron microscopy may lead the way towards new thinking about problems of transport by demonstrating that lipid droplets can seemingly penetrate the cell membrane.

\section{SUMMARY}

Administration of linseed oil into the duodenum of rats in vivo has resulted in the electron microscopic demonstration of the uptake of dense droplets by the microvilli of the jejunal surface epithelium. The significance of fat transport through the microvilli is discussed.

I wish to thank Professor D. Lacy and the staff of the Electron Microscope Unit for their encouragement and help in the problems and technique of electron microscopy. Grateful thanks are also due to Dr. E. N. Rowlands and Dr. H. S. Wiggins for their advice in the preparation of this paper. Finally, I wish to acknowledge the help of Mrs. I. Prentice, Mr. A. Booker, and Miss M. Salway in preparing the diagram and in the printing of the micrographs, and Mrs. E. E. Cox for her assistance.

\section{REFERENCES}

Ashworth, C. T., Stembridge, V. A., and Sanders, E. (1960). Lipid absorption, transport and hepatic assimilation studied with electron microscopy. Amer. J. Physiol., 198, 1326-1328.

Borgström, B. (1964). Abstract. In Abstracts of Communications presented at the 1 st meeting of the Federation of European Biochemical Societies, A 125, p. 96. Academic Press, London.

Danielli, J. F. (1964). Lipid transport across cell membranes. In Proceedings of an International Symposium on Lipid Transport, edited by H. C. Meng, pp. 104-115. Thomas, Springfield, Ill.

Lacy, D.. and Taylor, A. B. (1962). Fat absorption by epithelial cells of the small intestine of the rat. Amer. J. Anat., 110, 155-185.

Ladman, A. J., Padykula, H. A., and Strauss, E. W. (1963). A morphological study of fat transport in the normal human jejunum. lbid., 112, 389-419.

Luft, J. H. (1961). Improvements in epoxy resin embedding methods. J. biophys. biochem. Cytol., 9, 409-414.

McNabb, J. D., and Sandborn, E. (1964). Filaments in the microvillous border of intestinal cells. J. Cell Biol., 22, $701-704$.

Millington, P. F. (1964). Comparison of the thicknesses of the latera wall membrane and the microvillous membrane of intestinal epithelial cells from rat and mouse. Ibid., 20, 514-517.

-_, and Finean, J. B. (1962). Electron microscope studies of the structure of the microvilli on principal epithelial cells of rat jejunum after treatment in hypo- and hypertonic saline. Ibid., 14, 125-139.

Palay, S. L., and Karlin, L. J. (1959). An electron microscopic study of the intestinal villus. I. The fasting animal. II. The pathway of fat absorption. J. biophys. biochem. Cytol., 5, 363-384.

Proceedings of an International Symposium on Lipid Transport, edited by H. C. Meng, pp. 33-43. Thomas, Springfield, IIl.

Parsons, D. S. (1963). Quantitative aspects of pinocytosis in relation to intestinal absorption. Nature (Lond.), 199, 11929 $\mathcal{\varepsilon} .-11$

Reynolds, E. S. (1963). The use of lead citrate at high $p \mathrm{H}$ as an electron-opaque stain in electron microscopy. J. Cell Biol., 17, 208-211.

Rostgaard, J., and Barrnett, R. J. (1964). Fat absorption as micelles by microvilli of the small intestine in rats. In $3 r d$ European Regional Conference on Electron Microscopy, pp. 433-434. Czec.losl ovak Acadeny of Sciences, Prague.

Sabatini, D. D., Bensch, K., and Barrnett, R. J. (1963). Cytochemistry and electron microscopy. The preservation of cellular ultrastructure and enzymatic activity by aldehyde fixation. J. Cell Biol., 17, 19-58.

Shiner, M., Lacy, D., and Hudson, R. H. (1962). Electron micrcscope study of fat absorption in normal subjects and in patients with idiopathic steatorrhoea. In Intestinal Biopsy. Ciba Foundation Study Group No. 14, edited by G. E. W. Wolstenholme and M. P. Cameron, pp. 24-38. Churchill, London.

Sjöstrand, F. (1963). The fine structure of the columnar epithelium of the mouse with special reference to fat absorption. In Biochemical Problems of Lipids, edited by A. C. Frazer, pp. 91-115. Elsevier, New York.

Watson, M. L. (1958). Staining of tissue sections for electron microscopy wiih heavy metals. J. biophys. biochem. Cytol., 4, 475478. 